\title{
Placement of Distributed Energy Storage via Multidimensional Scaling and Clustering
}

\author{
Arthur K. Barnes \\ Department of Electrical Engineering \\ University of Arkansas \\ Fayetteville, AR USA \\ artbarnes@ieee.org
}

\author{
Juan Carlos Balda \\ Department of Electrical Engineering \\ University of Arkansas \\ Fayetteville, AR USA \\ jbalda@uark.edu
}

\begin{abstract}
Energy storage has long been proposed at the distribution level, where it can provide additional benefits via ancillary services. This work studies how to place energy storage units (ESU) on a distribution feeder in the most cost-effective manner while still meeting voltage regulation requirements. The feeder also has photovoltaic (PV) generation, and the PV ability to supply reactive power is considered. The placement of the ESU is performed via a fast heuristic, in which multidimensional scaling (MDS) is used to transform the combinatorial placement problem into a continuous-valued problem by mapping buses to points in a space. In the new space, clustering algorithms can be applied to determine the ESU locations from a set of candidate locations. The method reduces computation time by an order of magnitude, allowing for various distribution feeder configurations to be quickly compared.
\end{abstract}

Keywords-Energy storage, photovoltaic systems, renewable energy sources, optimization, clustering methods

\section{INTRODUCTION}

This paper focuses on ESU used principally for providing frequency regulation, but also for supplying reserve power during periods of high demand. The case addressed is a distribution system operator (DSO) that has made the purchase of a fixed amount of energy storage in terms of MWh capacity and "smart" PV inverters. The DSO seeks to determine the best solution in terms of numbers of ESU and "smart" PV units, their power ratings and placements on the system.

The "smart" PV inverter is a concept proposed by EPRI [1] that has the ability to draw or inject reactive power based on a power vs. voltage curve, allowing it to assist with voltage regulation. Because of the "smart" PV functions, it is desirable to integrate them with the ESU at the same time. When determining placement, the scenario of ESU and "smart" PV providing reserve power during peak load conditions is examined. The total load on the distribution feeder as viewed from the distribution substation can be lowered by appropriately placing the ESU and PV to flatten the voltage profile on the feeder, thereby allowing for conservation voltage reduction (CVR) to be applied [2]. CVR refers to lowering the overall voltage on the system, (usually by the substation transformer tap settings), in order to reduce overall load demand by taking advantage of the increasing power vs.

The authors are grateful for the financial assistance by the industry members of Grid-Connected Advanced Power Electronics Systems, an NSF I/UCRC voltage curve of the system load. However, dispersing the ESU on a distribution feeder as opposed to placing them at the substation will impact their ability to provide frequency regulation services.

This placement problem is closely related to that of placing distributed generation and shunt capacitors. The existing methods for placing these devices are categorized as: analytical, numerical optimization, heuristic search, and randomized search. The most common approach is genetic algorithms (GA), a form of randomized search which comes in both pure and hybrid optimal power flow (OPF) forms [3]. In the latter, the GA only selects the ESU locations, while an inner OPF selects their required power ratings, reducing the size of the GA search space and improving convergence time.

The placement method proposed in this work is a heuristic search that takes advantage of the observation that the number of ESU to be placed is small when compared to the total number of buses in the distribution feeder. The placement problem is then converted from a combinatorial problem to a continuous-valued problem by transforming the ESU bus locations into points in a continuous space with MDS. The ESU positions are then calculated with a clustering algorithm in the new space. By contrast, it is assumed that the power ratings of the "smart" PV installations are low as is the case with rooftop installations, and many of them can be placed on the feeder.

Although hierarchical clustering algorithms exist that can operate over a pairwise distance matrix, they suffer from sensitivity to initial conditions. The MDS algorithm allows classical k-means type algorithms to be applied, which are much less sensitive [4]. Additionally, it comes at a low cost in terms of both computational and programming burden, as it can be implemented in two lines in a high-level language with a set of matrix multiplications and eigenvector decomposition.

Clustering for placement reduces computational complexity over both pure and hybrid GA approaches, because it only runs a computationally intensive OPF twice, as opposed to the $10 \mathrm{~s}$ to $100 \mathrm{~s}$ of OPF necessary for GA [5]. This enables a distribution feeder designer to quickly evaluate several different ESU/"smart" PV configurations in a hypothetical software application by clicking a toolbar button.

The remaining sections of this paper describe how the method operates, illustrate its application to place ESU on a distribution feeder, evaluate the benefits of the ESU, and compare its performance against an existing GA approach. 


\section{CAlculating CANDidAte LocAtions AND POWER INJECTIONS USING AN OPF}

The ESU inject power to increase frequency towards $60 \mathrm{~Hz}$ (referred to here as up-regulating) or supply power during critical peaks. They also draw power to help reduce frequency towards $60 \mathrm{~Hz}$ (referred to here as down-regulating) and charge during off-peak times to account for losses. Thus, while it is desirable to place ESU where they make a large impact on the voltage regulation and power drawn by the feeder during critical peak load reduction and up-regulation, this placement is a hindrance when the ESU are to downregulate or charge.

The problem objective is to minimize the cost of power delivered from the substation bus during periods of high demand, expressed as

$$
\min . \sum_{t=1}^{N_{t}} C_{t} P_{1 t},
$$

where $t$ is the index of the current load/ESU/PV scenario, $N_{t}$ is the number of scenarios, $P_{1 t}$ is the power supplied by the substation bus (bus 1) during the current scenario, and $C_{t}$ is the relative cost of electricity supplied during the current scenario.

The problem is subject to several constraints, which are discussed in the remainder of this section. It is assumed that the ratio of the total power rating to the total energy capacity of the ESU is fixed at approximately 1:1 [6]. This means that the total energy capacity in MWh of energy storage purchased is equal to the total power rating of all ESU in MVA. Thus,

$$
\sum_{n=1}^{N} S_{n}^{e s u} \leq S_{\text {tot }}^{e s u}
$$

where $N$ is the number of buses, $S_{n}^{e s u}$ is the rated power of the ESU at bus $n$, and $S_{\text {tot }}^{e s u}$ is the total rated power of all the ESU in pu. For an optimal solution, the two terms in (2) will be equal. Similarly,

$$
\sum_{n=1}^{N} S_{n}^{p v} \leq S_{t o t}^{p v}
$$

where $S_{n}^{p v}$ is the rated power of the PV inverter at bus $n$ and $S_{t o t}^{p v}$ is the total rated power of all the PV inverters in MVA.

The apparent power scaling is a vector whose length is the number of scenarios included in the problem, where each element $K_{t}^{e s u}$ represents the fraction of rated power that each ESU operates at during the corresponding scenario. An equivalent element $K_{t}^{p v}$ applies for the PV. The power scalings for ESU and PV are not included as decision variables because these quantities are typically determined by a scheduler independent of network constraints; this assumption represents current practice [7]. Similarly, power factors for ESU and PV are also not included.

Rather than having these quantities as decision variables, they are used as inputs to the problem. The power factors are not represented directly, but instead as the angle between voltage and current. For the ESU and PV, these are represented as $\phi_{n t}^{e s u}$ and $\phi_{n t}^{p v}$, respectively. The real and reactive powers for the ESU and PV are given by

$$
\begin{aligned}
P_{n t}^{e s u} & =K_{t}^{e s u} S_{n}^{e s u} \cos \phi_{t}^{e s u} \\
Q_{n t}^{e s u} & =K_{t}^{e s u} S_{n}^{e s u} \sin \phi_{t}^{e s u} \\
P_{n t}^{p v} & =K_{t}^{p v} S_{n}^{p v} \cos \phi_{t}^{p v} \\
Q_{n t}^{e s u} & =K_{t}^{p v} S_{n}^{p v} \sin \phi_{t}^{p v} .
\end{aligned}
$$

The cost of installing the ESU is

$$
c=\sum_{n=1}^{N}\left(c^{\prime} S_{n}^{e s u}+c_{0} u_{n}\right),
$$

where $c$ is the total cost of placing the ESU in U.S. dollars, $c^{\prime}$ is the incremental cost of placing an ESU in dollars per pu, $c_{0}$ is the fixed installation cost of placing an ESU, and $u_{n}$ is a binary variable indicating if an ESU is placed at bus $n$.

These quantities are not used again, except to highlight that the cost of installing each ESU at bus $n$ consists of a fixed installation cost $\left(c_{0}\right)$ plus a component that depends on the capacity of the ESU $\left(c^{\prime}\right)$. The effect of this piecewise linear cost function is that it is more cost-effective to have a smaller number of larger ESU, as less fixed installation cost is incurred. In this study, the PV does not have any such fixed cost, and there is no incentive to aggregate PV installations.

Including a nonlinear cost makes the problem considerably harder, since it now has a mixed-integer nonlinear formulation [3]. In order to circumvent this issue, the OPF formulation is only used to select candidate buses for ESU placement. The placement is solved as a separate problem, using the candidate buses and the necessary power injections as inputs to a clustering algorithm which determines the actual ESU power ratings and locations. The clustering algorithm requires that the number of ESU be selected beforehand. However, as this number is small, it is reasonable to determine its optimal value by an exhaustive iteration over a fixed range from 0 to a maximum number of ESU $N_{\text {max }}^{e s u}$.

Using a static load model, which is discussed later in more detail, the power injections into each bus are represented as

$$
\begin{aligned}
P_{n t} & =P_{n t}^{e s u}+P_{n t}^{p v}-P_{n t}^{d} \\
Q_{n t} & =Q_{n t}^{e s u}+Q_{n t}^{p v}-Q_{n t}^{d} \\
P_{n t}^{d} & =K_{t}^{d} P_{n}^{d 0}\left|V_{n t}\right|^{\beta_{p}} \\
Q_{n t}^{d} & =K_{t}^{d} Q_{n}^{d 0}\left|V_{n t}\right|^{\beta} .
\end{aligned}
$$

In the above, $P_{n t}$ and $Q_{n t}$ are the real and reactive powers supplied by bus $n$ during scenario t. $P_{n t}^{d}$ and $Q_{n t}^{d}$ are the load real and reactive powers for bus $n . P_{n}^{d 0}$ and $Q_{n}^{d 0}$ are the rated load real and reactive power demands for bus $n$. $K_{t}^{d}$ is the feeder loading during the current scenario $t . V_{n t}$ is the voltage at bus $n$ during scenario $t$. The exponents $\beta_{p}$ and $\beta_{q}$ represent the relationship between voltage and load power, discussed in more detail later. The power flow constraints are represented as

$$
\begin{gathered}
P_{n t}=\left(P_{n t}^{e s u}+P_{n t}^{p v}-P_{n t}^{d}\right) \\
-\sum_{m=1}^{N}\left|V_{n t}\right|\left|V_{m t}\right| y_{m n} \cos \left(\theta_{m n}-\delta_{m t}-\delta_{n t}\right) \\
Q_{n t}=\left(P_{n t}^{e s u}+P_{n t}^{p v}-P_{n t}^{d}\right) \\
-\sum_{m=1}^{N}\left|V_{n t}\right|\left|V_{m t}\right| y_{m n} \sin \left(\theta_{m n}-\delta_{m t}-\delta_{n t}\right) \\
S_{n}^{e s u} \leq S_{m a x}^{e s u}
\end{gathered}
$$




$$
\begin{gathered}
V_{\text {min }} \leq\left|V_{n t}\right| \leq V_{\text {max }} \\
V_{\text {max }}=1.05 V_{\text {base }} \\
V_{\text {min }}=0.95 V_{\text {base }} .
\end{gathered}
$$

In the above, $y_{m n}$ is the magnitude of complex impedance matrix $\mathbf{Y}$, while $\theta_{m n}$ is the phase of the impedance between buses $m$ and $n$. The variable $\delta_{n t}$ is the phase of the voltage at bus $n$ during scenario $t$. $V_{\min }$ and $V_{\max }$ are the minimum and maximum allowable voltage magnitudes on the feeder respectively, while $V_{\text {base }}$ is the base voltage.

For this OPF, this exponential load model is used

$$
\begin{aligned}
& P_{n t}^{d}=K_{t}^{d} P_{n}^{d 0}\left|V_{n t}\right|^{\beta_{p}} \\
& Q_{n t}^{d}=K_{t}^{d} Q_{n}^{d 0}\left|V_{n t}\right|^{\beta_{q}} .
\end{aligned}
$$

In the above, $P_{n t}^{d}$ and $Q_{n t}^{d}$ are the load real and reactive power demands. The term $\left|V_{n t}\right|$ is the voltage magnitude in pu seen by the load. The terms $\beta_{p}=1.38$ and $\beta_{q}=3.32$ [8]. Again, $P_{n t}^{d 0}$ and $Q_{n t}^{d 0}$ are the rated real and reactive power demands of the load at a voltage of $1 \mathrm{pu}$, while $K_{t}^{d}$ is the feeder loading during the current scenario $t$. In order to calculate the required amount of power injection at each candidate bus, a nonlinear constrained optimizer is used on the problem, in this case fmincon () from the MATLAB ${ }^{\mathrm{TM}}$ optimization toolbox.

\section{SELECTION OF THE ESU LOCATIONS}

In this particular problem, the key idea is that it is not desirable to place ESU at buses that are very "close" to one another, which in this context means that the impedance between them is low. To determine how to best combine the candidate locations into a smaller number of well-spaced ESU, the candidate locations are mapped into a continuous space that is easier to work with than the original discrete locations. To accomplish this, dimensionality scaling methods are employed [9], [10]. However, even with the candidate locations mapped into a continuous space, the problem is still combinatorial in nature. Fortunately, the new problem space allows for the use of clustering methods, which represent a "good" suboptimal solution to the problem [4].

\section{A. Multidimensional Scaling}

The candidate buses are mapped to points in a $q$ dimensional space using MDS, a dimensionality reduction technique that is commonly used in the social sciences. It works by first calculating pairwise distances between each object (in this case, a bus) in a set (in this case, the $M$ ESU candidate locations). The objects are then mapped to arbitrarily placed points in the $q$-dimensional space. The classical MDS (CMDS) algorithm is employed. It maps the objects using their pairwise distances onto a $q$-dimensional space. It does this by applying a pair of transformation matrices in order to convert the pairwise distance matrix $\mathbf{D}$ into a positive semidefinite matrix $\mathbf{B}$ of rank $q$. The matrix $\mathbf{B}$ is then used to produce matrix $\mathbf{W}$, whose columns are the position vectors $\boldsymbol{w}_{1}, \boldsymbol{w}_{2}, \ldots, \boldsymbol{w}_{M}$.

The method is illustrated as follows:

First, the distance matrix $\mathbf{D}$ is transformed

$$
\mathbf{B}=\left(\mathbf{I}_{M}-M^{-1} \mathbf{1}_{M} \mathbf{1}_{M}^{T}\right) \mathbf{A}\left(\mathbf{I}_{M}-M^{-1} \mathbf{1}_{M} \mathbf{1}_{M}^{T}\right) .
$$

In the above expression,

$$
\begin{gathered}
a_{n m}=\frac{1}{2} d_{n m} \\
\mathbf{1}_{M}=\left[\begin{array}{llll}
1 & 1 & \cdots & 1
\end{array}\right]^{T},
\end{gathered}
$$

where $a_{n m}$ and $d_{n m}$ denote the elements of in the $n^{\text {th }}$ row and $m^{\text {th }}$ column of the matrices $\mathbf{A}$ and $\mathbf{D}$, respectively. The term $\mathbf{I}_{M}$ is a $M \times M$ identity matrix. The $q$ eigenvectors $\boldsymbol{v}_{n}$ corresponding to the nonzero eigenvalues $\lambda_{n}$ of $\mathbf{B}$ are selected, i.e. those corresponding to

$$
\mathbf{B} \boldsymbol{v}_{n}=\lambda_{n} \boldsymbol{v}_{n}, \quad \lambda_{n} \neq 0
$$

These are concatenated into a new matrix

$$
\mathbf{W}=\left[\boldsymbol{v}_{1}, \boldsymbol{v}_{2}, \ldots, \boldsymbol{v}_{q}\right]^{T} .
$$

Each column $\boldsymbol{w}_{n}$ of $\mathbf{W}$ represents a point in the new space.

The essence of the method is that if each element of $\mathbf{A}, a_{n m}$ is proportional to a distance metric $d_{n m}$ calculated between objects $n$ and $m$, then the Euclidean distance between columns $\boldsymbol{w}_{n}$ and $\boldsymbol{w}_{m}$ of $\mathbf{W}$ is also $d_{m n}$. Thus, by the definition of an Euclidean distance,

$$
d_{n m}=\left\|\boldsymbol{w}_{n}-\boldsymbol{w}_{m}\right\|_{2} .
$$

It is important to realize however, that the distances, $d_{n m}$, do not necessarily correspond to the distances between two points in an original space, as is the case with both similarity scores on questionnaires and the impedance between two buses in a distribution feeder.

\section{B. Clustering Algorithm}

The goal of the clustering algorithm is to group together buses that are candidate locations. To perform the clustering, a number of clusters $K$ (corresponding to the number of ESU to be placed) is selected as an input to the clustering algorithm. The requirements of the clustering algorithm are to assign each bus to a particular ESU and to select the bus that the units will be placed at. Hence, the objective of the clustering algorithm is to minimize the intra-cluster variance

$$
J=\sum_{n \in \mathcal{D}_{k}}\left\|\boldsymbol{w}_{n}-\boldsymbol{\mu}_{k}\right\|^{2}
$$

where $\boldsymbol{w}_{n}$ is a point in cluster $k, \boldsymbol{\mu}_{k}$ is the centroid (or center of mass) of cluster $k$, and $\mathcal{D}_{k}$ is the set of points belonging to cluster $k$. A last issue is that the amount of ESU power injection at each bus is not identical. This could result in erroneous results if there is a bus with only a small amount of power injection and another bus with a large amount. To account for this, the k-means algorithm is used [4], but the centroid calculation is modified to use the amount of power injection at each bus as a weight. In the original k-means algorithm, the centroids are calculated as

$$
\boldsymbol{\mu}_{k}=\frac{1}{N_{k}} \sum_{n \in \mathcal{D}_{k}} \boldsymbol{w}_{n},
$$

where $N_{k}$ is the number of points in cluster $k$. By contrast, in the modified algorithm, the centroids are calculated as

$$
\boldsymbol{\mu}_{k}=\sum_{n \in \mathcal{D}_{k}} \gamma_{n} \boldsymbol{w}_{n},
$$

where the weighting $\gamma_{n}$ is equal to 


$$
\gamma_{i}=\frac{S_{n}^{e s u}}{\sum_{n \in \mathcal{D}_{k}} S_{n}^{e s u}} .
$$

To perform placement, the ESU are allocated to the bus nearest to their corresponding centroid. The power injections of each ESU candidate are then assigned to their corresponding ESU, as illustrated in Fig. 1.

\section{CASE StUdy AND Numerical Results}

The proposed method is tested on the radial Italian rural distribution feeder given in [11] and illustrated in Fig. 2. There are two substations at buses 1 and 2, the load profile is shown in Fig. 3, and the parameters are listed in Tables I and II.

The total power rating of all the ESU to be placed is set at $1 / 10^{\text {th }}$ the total feeder real power demand, while the total power rating of the "smart" PV to be placed is $1 / 40^{\text {th }}$ the total feeder real power demand. Based on the results in [11], the number of ESU to be placed is 2. This is presented in Table III, along with other relevant inputs to the placement problem.

Five different scenarios were analyzed in placement. In addition to the base case without ESU or PV, a peak evening condition was selected for the objective. The ESU and PV are placed to minimize power demand from the feeder under this condition. As an evening case is used, the PV injects reactive power only. In addition, three other cases are included, which verify if the ESU is able to provide frequency regulation without causing flicker issues, as well as its ability to charge and discharge without violating voltage constraints. These scenarios are presented in Tables IV and V.

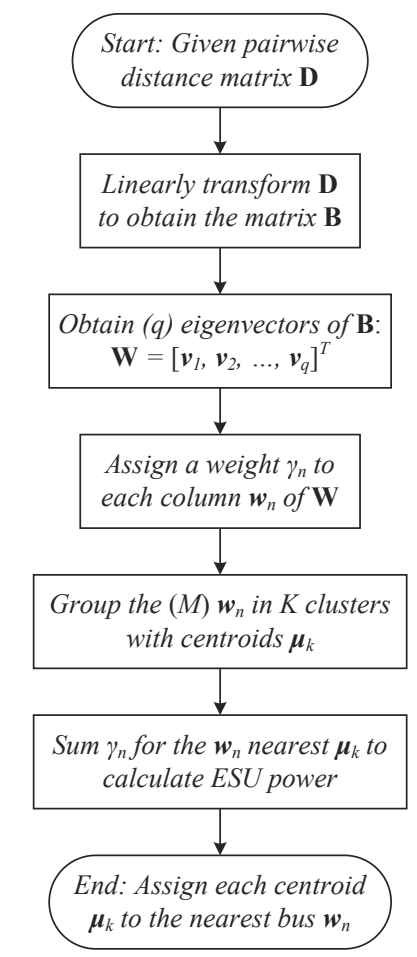

Fig. 1. Flowchart for the CMDS/clustering algorithm.

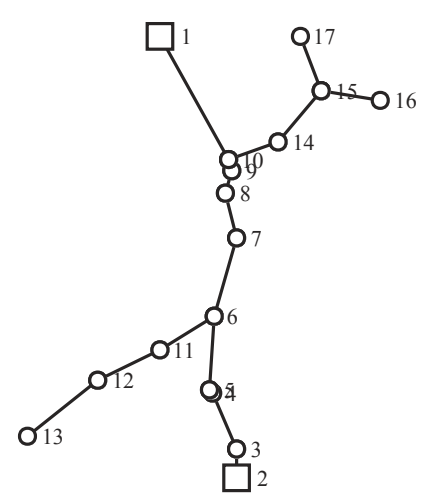

Fig. 2. Configuration of the feeder case study.

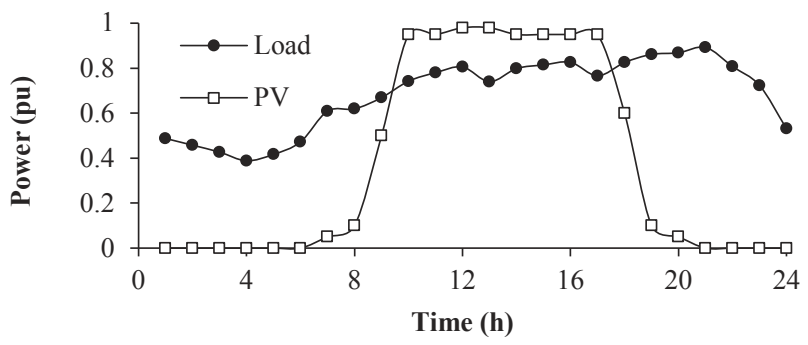

Fig. 3. Feeder average load and PV profile over one day.

TABLE I. BRANCH CONNECTIVITY AND IMPEDANCES

\begin{tabular}{rrcc}
\hline From & To & $\mathbf{R}$ & $\mathbf{X}$ \\
\hline 1 & 10 & 0.00169 & 0.00073 \\
10 & 14 & 0.00676 & 0.00291 \\
14 & 15 & 0.00024 & 0.00010 \\
15 & 16 & 0.00507 & 0.00218 \\
15 & 17 & 0.00566 & 0.00268 \\
10 & 9 & 0.00338 & 0.00146 \\
9 & 8 & 0.00217 & 0.00094 \\
8 & 7 & 0.00048 & 0.00021 \\
7 & 6 & 0.00435 & 0.00187 \\
6 & 5 & 0.00483 & 0.00208 \\
5 & 4 & 0.00580 & 0.00250 \\
4 & 3 & 0.00386 & 0.00166 \\
3 & 2 & 0.00531 & 0.00229 \\
6 & 11 & 0.00435 & 0.00187 \\
11 & 12 & 0.00435 & 0.00187 \\
12 & 13 & 0.00966 & 0.00416 \\
\hline
\end{tabular}


TABLE II. LOAD POWER AT EACH Bus

\begin{tabular}{ccc}
\hline Bus Number & Power $(\mathbf{k W})$ & Power Factor \\
\hline 1 & 0.00 & 0.00 \\
2 & 0.00 & 0.00 \\
3 & 90.00 & 0.90 \\
4 & 100.00 & 0.90 \\
5 & 320.00 & 0.90 \\
6 & 90.00 & 0.90 \\
7 & 83.67 & 0.90 \\
8 & 420.00 & 0.90 \\
9 & 60.00 & 0.90 \\
10 & 90.00 & 0.90 \\
11 & 0.00 & 0.00 \\
12 & 600.00 & 0.88 \\
13 & 50.00 & 0.71 \\
14 & 550.00 & 0.71 \\
15 & 500.00 & 0.90 \\
16 & 400.00 & 0.90 \\
17 & 0.00 & 0.00 \\
\hline
\end{tabular}

TABLE III. CASE Study Parameters

\begin{tabular}{ccc}
\hline Parameter & Expression & Value \\
\hline Base Voltage & $V_{\text {base }}$ & $20 \mathrm{kV}$ \\
Base Power & $S_{\text {base }}$ & $2 \mathrm{MW}$ \\
$\begin{array}{c}\text { Substation 2 } \\
\text { power } \\
\text { Number of } \\
\text { ESU }\end{array}$ & $P_{2 t}, Q_{2 t}$ & $\frac{K_{t}^{d}}{6} \sum_{n=1}^{N} P_{n}^{d 0}, \frac{1}{6} \sum_{n=1}^{N} Q_{n}^{d 0}$ \\
Total ESU & $K$ & 2 \\
Power & $S_{\text {tot }}^{e s u}$ & $\frac{1}{10} \sum_{n=1}^{N} P_{n}^{d 0}$ \\
Total PV Power & $S_{\text {tot }}^{\text {pv }}$ & $\frac{1}{40} \sum_{n=1}^{N} P_{n}^{d 0}$ \\
Minimum & $V_{\text {min }}$ & 0.95 pu \\
Voltage \\
Maximum \\
Voltage & $V_{\text {min }}$ & 1.05 pu \\
Cost & $C_{t}$ & $1, \quad t=1$ \\
\hline
\end{tabular}

TABLE IV. DESCRIPTION OF EVALUATED SCENARIOS

\begin{tabular}{cccc}
\hline Scenario & Load State & PV State & ESU State \\
\hline 0 (base) & Peak & Off & Off \\
1 (objective) & Peak & Inject Q & Discharge \\
2 (up-regulating) & Peak & Off & Discharge \\
3 (down-regulating) & Peak & Off & Charge \\
4 (up-regulating) & Off-Peak & Off & Discharge \\
\hline
\end{tabular}

TABLE V. LOAD, ESU AND PV PARAMETERS FOR EACH SCENARIO

\begin{tabular}{cccccc}
\hline Scenario & $K_{t}^{d}$ & $K_{t}^{e s u}$ & $\phi_{t}^{e s u}$ & $K_{t}^{p v}$ & $\phi_{t}^{p v}$ \\
\hline 0 (base) & 1.0 & 0.0 & 0 & 0 & 0.00 \\
1 (objective) & 1.0 & 1.0 & 0 & 1 & $\pi / 2$ \\
2 (up-regulating) & 1.0 & 1.0 & 0 & 0 & 0.00 \\
3 (down-regulating) & 1.0 & 1.0 & $\pi$ & 0 & 0.00 \\
4 (up-regulating) & 0.454 & 1.0 & 0 & 0 & 0.00 \\
\hline
\end{tabular}

Although the average feeder load does not reach $1.0 \mathrm{pu}$, a $1.0 \mathrm{pu}$ peak load is selected as a worst case. To evaluate whether flicker is an issue, the maximum change in voltage

$$
\Delta V=\max _{n}\left(\left|V_{n 2}\right|-\left|V_{n 3}\right|\right)
$$

at any bus is measured between scenarios 2 (up-regulating) and 3 (down-regulating). Assuming a 4-s period in the regulation signal, any change lower than $1 \%$ is considered tolerable according to the worst case for the IEC-868 flicker curve [12], [13]. To evaluate if voltage regulation problems are caused during charging and discharging, scenarios 3 (peak load, upregulating) and 4 (off-peak load, down-regulating) are studied.

To evaluate the performance improvement against competing methods, the placement problem is also performed with genetic algorithms, following the approach of the original analysis [11]. To more fairly compare against the proposed method, the chromosome encoding is changed so that the number of ESU to be placed is fixed, as illustrated in Fig. 4. Rather than encoding the ESU placement as a binary vector whose number of elements is the number of buses $N$, it is now encoded as an integer vector whose number of elements is the number of ESU to be placed $K$. Additionally, the parameters listed in Table VI are applied.

Tables VII-IX present placement results. The genetic algorithm was able to find the optimal placement after the first generation because of the small search space. However, the clustering-based placement is able to complete in under $2 \mathrm{~s}$, over an order of magnitude less than a single iteration of the genetic algorithm. The results for the different scenarios indicate that the optimal placement of the ESU for reducing peak power does not cause voltage violations under other conditions. However, the ability of the ESU to provide regulation services is impeded, as the worst-case voltage variation will be noticeable.

TABLE VI. Genertic Algorithm PARAMETERS

\begin{tabular}{cc}
\hline Parameter & Value \\
\hline Fitness function & rank \\
Selection function & remainder selection \\
Population replacement & generational \\
& $\frac{1}{20}$ min $[\max (10 N, 40), 100]$ \\
Elite individuals & $(2-5$ depending on sample size $)$ \\
& uniform \\
Mutation function & 0.01 \\
Mutation rate & scattered \\
Crossover type & 0.8 \\
Crossover fraction & $\max (N, 30)$ \\
Population size & 100 \\
Convergence iterations & 200 \\
Maximum iterations & \\
\hline
\end{tabular}




\begin{tabular}{|c|c|c|c|}
\multicolumn{1}{c}{} & \multicolumn{2}{c}{$K$} \\
\hline $1, \ldots, N$ & $1, \ldots, N$ & $\ldots$ & $1, \ldots, N$ \\
\hline
\end{tabular}

Fig. 4. Chromosome encoding for the genetic algorithm.

TABLE VII. Clustering Placement Results

\begin{tabular}{cc}
\hline Parameter & Value \\
\hline ESU buses & 13,16 \\
ESU power ratings $(\mathrm{kW})$ & $127.8,207.6$ \\
PV total power rating $(\mathrm{kW})$ & 83.84 \\
Feeder demand without PV and ESU (MW) & 3.174 \\
Feeder demand with PV and ESU (MW) & 2.898 \\
Reduction in feeder demand $(\mathrm{kW})$ & 611.1 \\
Max $\Delta V$ between charge and discharge $(\%)$ & 1.333 \\
Clustering time to complete $(\mathrm{s})$ & 2.073 \\
\hline
\end{tabular}

TABLE VIII. GA PlaCEMENT RESUlts

\begin{tabular}{cc}
\hline Parameter & Value \\
\hline ESU buses & 12,16 \\
ESU power ratings $(\mathrm{kW})$ & $175.3,160.1$ \\
PV total power rating $(\mathrm{kW})$ & 83.84 \\
Feeder demand with PV and ESU (MW) & 2.985 \\
Reduction in feeder demand (kW) & 614.7 \\
Generations to convergence & 1 \\
Time to complete & $35 \mathrm{~s} /$ iteration \\
\hline
\end{tabular}

TABLE IX. OPF Results For Clustering Placement

\begin{tabular}{cccc}
\hline Scenario & $\begin{array}{c}\text { Feeder } \\
\text { Power }\end{array}$ & $\begin{array}{c}\text { Min. } \\
\text { Voltage }\end{array}$ & $\begin{array}{c}\text { Max. } \\
\text { Voltage }\end{array}$ \\
\hline 0 (base) & 3.1741 & 0.9500 & 0.9976 \\
1 (objective) & 2.8984 & 0.9500 & 0.9928 \\
2 (up-regulating) & 2.8027 & 0.9500 & 0.9912 \\
$\begin{array}{c}\text { 3 (down- } \\
\text { regulating) }\end{array}$ & 3.5534 & 0.9500 & 1.0046 \\
4 (up-regulating) & 1.0096 & 0.9500 & 0.9639 \\
\hline
\end{tabular}

\section{CONCLUSIONS}

A placement method for distributed ESU that considers the addition of "smart" PV inverters was proposed. It demonstrated the utility of ESU in reducing peak load, while quantifying the amount of unwanted flicker introduced by performing frequency regulation with the placed ESU. The method employed a novel heuristic involving MDS and clustering that reduced computation time compared to the genetic algorithm methods commonly applied to this class of problems. This reduced computational time makes the method appealing for use in interactive software packages such as distribution analysis applications.

\section{REFERENCES}

[1] B. Seal, "Specification for smart inverter interactions with the electric grid using international electrotechnical commission 61850," Electric Power Research Institute, Knoxville, TN, Standard 1021674, Oct. 2010.

[2] C. A. McCarthy and J. Josken, "Applying capacitors to maximize benefits of conservation voltage reduction," in Rural Electric Power Conference, 2003, pp. C4-1- C4-5.

[3] J. C. Carlisle, A. A. El-Keib, D. Boyd, and K. Nolan, "A review of capacitor placement techniques on distribution feeders," in TwentyNinth Southeastern Symposium on Syst. Theory, 1997, pp. 359-365.

[4] R. O. Duda, P. E. Hart, and D. G. Stork, Pattern Classification. Wiley, 2001 .

[5] A. Alarcon-Rodriguez, E. Haesen, G. Ault, J. Driesen, and R. Belmans, "Multi-objective planning framework for stochastic and controllable distributed energy resources," IET Renew. Power Gener., vol. 3, no. 2, pp. 227-238, Jun. 2009

[6] M. T. Holmberg, M. Lahtinen, J. McDowall, and T. Larsson, "SVC Light ${ }^{\circledR}$ with energy storage for frequency regulation," in IEEE Conference on Innovative Technologies for an Efficient and Reliable Electricity Supply (CITRES), 2010, pp. 317-324.

[7] A. Barnes, J. C. Balda, A. Escobar, and S. O. Geurin, "Optimal battery chemistry, capacity selection, charge/discharge schedule, and lifetime of energy storage under time-of-use pricing," in IEEE PES ISGT Europe, Manchester, England, 2011.

[8] M. E. El-hawary and L. G. Dias, "Incorporation of load models in loadflow studies. form of model effects," Proc. IEEE Gener. Transm. Distrib., vol. 134, no. 1, pp. 27-30, Jan. 1987.

[9] F. Belmudes, D. Ernst, and L. Wehenkel, "Pseudo-geographical representations of power system buses by multidimensional scaling," in Int. Conf. on Intelligent Syst. Appl. to Power Syst., 2009, pp. 1-6.

[10] G. Yesuratnam and D. Thukaram, "Congestion management in open access based on relative electrical distances using voltage stability criteria," Electr. Power Syst. Res., vol. 77, no. 12, pp. 1608-1618, Oct. 2007.

[11] G. Celli, S. Mocci, F. Pilo, and M. Loddo, "Optimal integration of energy storage in distribution networks," in IEEE PowerTech, Burcharest, 2009, pp. 1-7.

[12] R. C. Dugan, M. F. McGranaghan, S. Santoso, and H. W. Beaty, Electrical Power Systems Quality, Third Edition. McGraw Hill Professional, 2012.

[13] N. Lu, M. R. Weimar, Y. V. Makarov, F. J. Rudolph, S. N. Murthy, J. Arseneaux, and C. Loutan, "Evaluation of the flywheel potential for providing regulation service in California," in 2010 IEEE Power and Energy Society General Meeting, 2010, pp. 1-6. 